\title{
Probing the Influence of Multiscale Heterogeneity on Effective Properties of Graphite Electrodes
}

Chance Norris ${ }^{1}$, Mukul Parmananda ${ }^{1}$, Scott A. Roberts ${ }^{2,}$, and Partha P. Mukherjee ${ }^{1, *}$

${ }^{1}$ School of Mechanical Engineering, Purdue University, West Lafayette, IN 47907, USA

${ }^{2}$ Engineering Sciences Center, Sandia National Laboratories, Albuquerque, New Mexico 87185, USA

*Correspondence: sarober@sandia.gov (S. A. Roberts); pmukherjee@purdue.edu (P.P. Mukherjee) 


\begin{abstract}
Graphite electrodes in the lithium-ion battery exhibit various particle shapes, including spherical and platelet morphologies, which influence structural and electrochemical characteristics. It is well established that porous structures exhibit spatial heterogeneity, and the particle morphology can influence transport properties. The impact of the particle morphology on the heterogeneity and anisotropy of geometric and transport properties has not been previously studied. This study characterizes the spatial heterogeneities of 18 graphite electrodes at multiple length scales by calculating and comparing the structural anisotropy, geometric quantities, and transport properties (pore-scale tortuosity and electrical conductivity). We found that the particle morphology and structural anisotropy play an integral role in determining the spatial heterogeneity of directional tortuosity and its dependency on pore-scale heterogeneity. Our analysis reveals that the magnitude of in-plane and through-plane tortuosity difference influences the multiscale heterogeneity in graphite electrodes.
\end{abstract}

Keywords: Li-ion battery; graphite electrode; multiscale heterogeneity; structural anisotropy; transport property 


\section{Introduction}

Secondary battery technology is abundant in all aspects of our lives. However, it is paramount to continue advancing the technology to satisfy the ever-increasing demand for energy, power density, and long-range capabilities in electric vehicles ${ }^{1,2}$. Graphite is the most widely used anode material due to its low cost, abundance, and chemical stability. However, graphite particles have varied particle shapes or morphologies, including platelet, spherical, flaky, and many shapes inbetween $^{3}$ that influence the structural characteristics and, therefore, the electrochemical performance of the electrode ${ }^{4,5}$.

Due to the inability to fully control particle packing, particle morphologies can influence structural characteristics in the electrode, such as tortuosity anisotropy and heterogeneity. Müller et al. studied the electrode heterogeneity using submicron X-ray computed tomography (XCT). In contrast, others have looked at various length scales using neutron diffraction and Raman spectroscopy ${ }^{6-8}$. Compared to scanning electron microscopy (SEM) ${ }^{9,10}$ the field of view (FOV) for an XCT is magnitudes greater, though at the cost of resolution and the ability to differentiate low atomic number materials. A large FOV is crucial when analyzing the effects of the particle morphology on the entire electrode; a larger FOV encapsulates trends at the electrode scale but still has enough resolution to see possible influences of the morphology at the particle scale. Imaging techniques identified that the stochastic nature of particle packing during manufacturing ${ }^{11}$ induces heterogeneity within electrode structures ${ }^{12,13}$. Such an electrode heterogeneity can cause local transport and reaction kinetics limitations within the electrode that could significantly influence the performance, safety, and aging behavior of lithium-ion cells significantly ${ }^{14-16}$. Heterogeneous packing of active material $1^{17,18}$ and varying particle morphology $19,20,17,21$ induces 
non-uniform performance, which results in localized lithium deposition ${ }^{22}$ and electrode degradation. ${ }^{23}$

Particle morphology is one of the main factors influencing particle orientation within an electrode, leading to anisotropy in the resulting electrode properties. Physics simulations have been executed on the electrode structures to explain how the particle morphology influences effective properties such as porosity, tortuosity, conductivity, and surface area ${ }^{6-8,24-28}$ These packed particle characteristics have been shown to directly influence anisotropies of effective properties ${ }^{18,21-24}$ from the particles' morphological anisotropies. These properties have a direct influence on the electrochemical performance of the cell..$^{27,29-31}$ Not only does the particles' morphological anisotropy influence structural anisotropy but we also show that these directly impact electrode heterogeneity. Therefore, it is essential to understand how these differing particle morpho logies will impact the cell's performance and safety.

With all of the research performed on electrode microstructures, none have dived into the effect of the particle morphology on structural heterogeneity and anisotropy. We use 18 commercial electrodes to analyze an extensive range of graphite morphologies. We study the correlations between the particle morphology and electrode heterogeneity. Using a combination of mean intercept length and finite volume physics simulations, we characterize the spatial hetero geneities at multiple length scales from the effects of the particle morphology. We calculate the tortuosity and electrical conductivity of 18 graphite electrodes imaged using XCT. ${ }^{20,32}$ Using multiple subdomains arranged in a grid over the entire electrode domain, we capture spatial variations in tortuosity, conductivity, and porosity over an electrode and between numerous samples from the same electrode sheet. We also characterize the particle morphology using the mean intercept length (MIL) to understand the connections between the morphology and heterogeneities. 


\section{Methodology}

We studied 18 graphite electrodes that were imaged by Wood and co-workers using XCT. ${ }^{20,32}$ We take their binarized data not to impose our subjectiveness in the segmentation process. ${ }^{33} \mathrm{We}$ classify these electrodes using either a numbering scheme (I, II, III, and IV), where the subscripts $\mathrm{a}, \mathrm{b}$, and $\mathrm{c}$ refer to electrodes taken from the same sheet at different locations, or a naming scheme, which denotes that the electrodes are from a particular manufacturer. These electrode identifications mirror those in the source papers..$^{20,32}$ We refer to these sets of electrodes as numbered and named for the remainder of the paper. All electrodes were taken from a commercial cylindrical cell and imaged, though the numbered samples do not specify the manufacturer. The numbered electrodes have a voxel dimension of $0.325 \mu \mathrm{m} \times 0.325 \mu \mathrm{m} \times 0.325 \mu \mathrm{m}$, while the named electrodes have a voxel dimension of $0.1625 \mu \mathrm{m} \times 0.1625 \mu \mathrm{m} \times 0.1625 \mu \mathrm{m}$. All images have identical $\mathrm{x}$ and $\mathrm{y}$ dimensions of $357.5 \mu \mathrm{m}$ and various $z$-directional thicknesses. We used the provided binarized image stacks for all electrodes. Figure 1 shows a schematic of each analysis step, all described in the remainder of this section. 


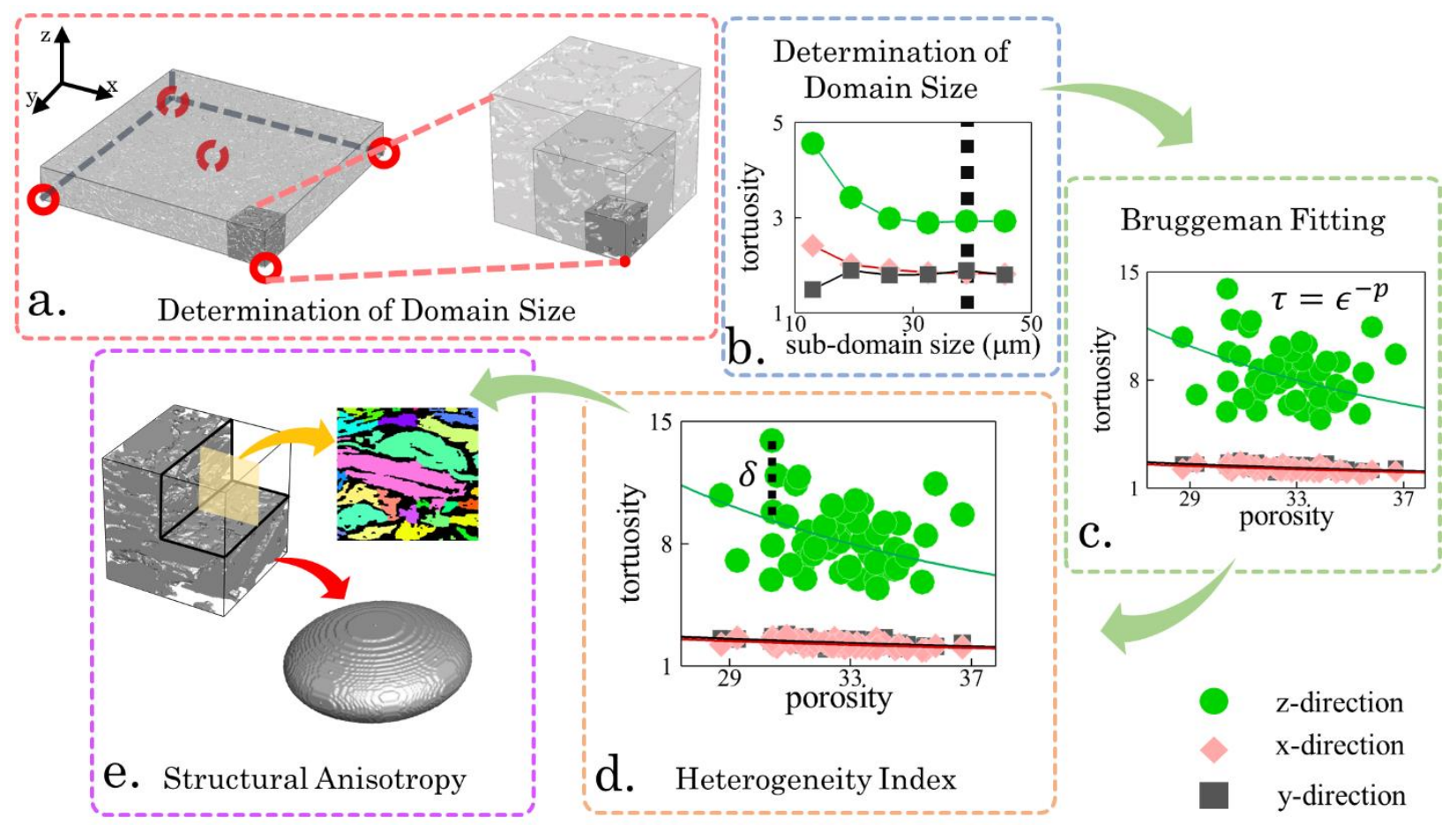

Figure 1. Steps to obtain the needed electrode information for analysis. $(a, b)$ Coarseness approximation of electrode IIc used to obtain an RVE size. Green, red, and black represent $z, x$, and $y$ directions, respectively. (c) Bruggeman correlation for tortuosity for electrode IVa. Each point shown represents an RVE and its directional tortuosity. (d) Heterogeneity index calculation. Comparing the actual value with the predicted value through the Bruggeman correlation. (e) Results from the mean intercept length algorithm to get the structural anisotropy.

\subsection{Effective Properties}

We calculate three effective properties to characterize electrochemical performance: porosity, tortuosity, and electrical conductivity. Each one has an integral part in electrode performance at the macroscale, influencing how lithium and electrons diffuse through phases and determine electrode capacity. $7,26,28,34$

Porosity is the volume fraction of the pore phase relative to the volume of the domain, as shown in eq 1:

$$
\epsilon=\frac{V_{\text {void }}}{V} .
$$


where $V_{\text {void }}$ is the volume of the void phase and $V$ is the entire domain's volume. Porosity is calculated computationally by summing the void voxels and dividing the result by the domain's total voxels.

Tortuosity, which gives insight into the pore phase connectivity, is calculated by solving for species diffusion within the pore phase:

$$
\nabla^{2} C=0 .
$$

where $C$ is the concentration and the boundary conditions in the $x$-direction for the normal faces ( $x=0$ and $x=L_{x}$, where $L_{x, y, z}$ is the voxel length in the given direction) are

$$
C(x=0)=0 \quad C\left(x=L_{x}\right)=1 .
$$

Neumann boundary conditions of no-flux are applied to the remaining faces $\left(z=0, y=0, z=L_{z}\right.$, $y=L_{y}$, and pore/particle boundaries). We solve these equations using the finite volume method on the voxelated mesh. We find tortuosity by integrating the species flux over the pore faces at $x=$ $L_{x}$, giving $J_{x}$, as shown in eq 4

$$
J_{x}=-\left.\int \frac{\delta C}{\delta x}\right|_{x=0} d y d z
$$

where $J_{x}$ is the species flux. Then, we obtain tortuosity using eq $526,35,36$

$$
\tau_{x}=-\frac{\epsilon}{J_{x}}\left(\frac{1}{L_{x}}\right)
$$


where $J_{x}$ is the species flux, $\tau_{x}$ is the $x$-direction tortuosity, $\varepsilon$ is the porosity, and $L_{x}$ is the voxel length in the $x$-direction.

The calculation for electronic conductivity has a similar approach taken in finding tortuosity. The governing equations occur throughout the particle phase

$$
\nabla \cdot(\zeta \nabla \phi)=0
$$

where $\varphi$ is the solid phase potential, the boundary conditions applied are identical to the tortuosity calculations. The electronic flux is calculated by eq 7

$$
J_{x}=-\left.\iint_{x=0 \text { plane }} \frac{\delta \phi}{\delta x}\right|_{x=0} d y d z
$$

Finally, the effective conductivity is shown in eq 8 , where $\sigma_{x}$ is the effective solid-phase electrical conductivity in the $x$-direction.

$$
\sigma_{x}=-J_{x} * L_{x}
$$

Calculating the tortuosity and electrical conductivity in the $y$ and $z$ dimensions uses the same approach but with the boundary conditions specifying the applied gradient to the corresponding faces. 


\subsection{Determination of Simulation Domain Size}

A representative volume element (RVE) is a subdomain of a structure representing the statistics of the entire domain. However, when studying heterogeneities within an electrode, we need to choose a simulation domain size, referred to as the local representative volume element (LRVE), large enough to represent the local phenomena statistically while not masking larger-scale electrode heterogeneities.

To determine the LRVE, we chose five subdomains origin locations from each electrode and iteratively created $3 \mathrm{D}$ subdomains sized from 13 to $45.5 \mu \mathrm{m}$ in $6.5 \mu \mathrm{m}$ increments. Then, we calculated the porosity, tortuosity, and electrical conductivity for each subdomain. An example of these simulations for the tortuosity of electrode IVa is shown in Figure 1(a). As the subdomains grow, the calculated values converge within themselves. We considered the sub domains converged when all effective property values varied less than $10 \%$ for at least three consecutive sub domain increments. Tortuosity and electrical conductivity are the slowest to converge and determine properties when choosing a domain size. The LRVE size of $39 \mu \mathrm{m} \times 39 \mu \mathrm{m} \times 39 \mu \mathrm{m}$ was the smallest domain where all properties converged for all electrodes and are, therefore, the sub domain size used for all electrodes in the remainder of this work. We look only at the localized regions individually rather than a holistic view used in a typical RVE. The through-plane directions converged last in most electrodes. Before $39 \mu \mathrm{m}$ edge lengths, the through-plane properties did not monotonically converge, meaning the LRVE size did not represent local through-plane tortuosity.

\subsection{Bruggeman Deviation}

The ability to compare the potential influences of heterogeneity across several particle morphologies is paramount to this study. Both tortuosity and conductivity in all three directions 
are individually fit to a standard Bruggeman approximation shown in equations 8 and 98,26,31,32,37, where each direction has a unique exponential value. We use these exponential values to probe for anisotropies comparing the ratios of the averaged in-plane to the through-plane exponents

$$
\tau_{x, y, z}=\epsilon^{-p_{x, y, z}} .
$$

We take a similar approach to find electrical conductivity. The modified Bruggeman approximation is shown as

$$
\sigma_{x, y, z}=(1-\epsilon)^{q_{x, y, z}} .
$$

where $(1-\varepsilon)$ is the solidity (ratio of the particle phase to domain volume). Equations 9 and 10 are fit to all LRVE values for individual properties and their respective directions.

We obtain the Bruggeman deviation $(\delta)$ by subtracting the predicted of the Bruggeman curves at the LRVE's porosity from the LRVE value of tortuosity or conductivity. The difference is then normalized with the Bruggeman predicted value, as shown in eq 11

$$
\delta_{L R V E}=\frac{\tau_{L R V E}-\tau_{\text {Bruggeman }}}{\tau_{\text {Bruggeman }}} .
$$

where $\delta_{L R V E}$ is the error of an individual LRVE, and we can compare electrodes with various magnitudes of tortuosity by normalizing the values. This procedure is performed on all electrodes, properties, and their respective direction. Averaging the errors for a given property and its 
direction, we get the Bruggeman deviation. The physical representation of the Bruggeman deviation is the average LRVE internal variability.

We use the exponent values from the Bruggeman fit to find effective property anisotropies from eq 12

$$
\text { anisotropy }_{\text {property }}=1-\frac{(p, q)_{\text {avg of } x, y}}{(p, q)_{z}} \text {. }
$$

We can describe effective property anisotropy by subtracting one from the ratio of the average in-plane directions to the through-plane. Anisotropy equal to 0 represents an isotropic structure, greater than 0 represents larger through-plane magnitude, and less than 0 represents larger in-plane magnitude.

\subsection{Structural Anisotropy}

The most direct approach in describing structural anisotropies $\left(\alpha_{s}\right)$ in porous structures analyzes the electrode's structure itself. We use the mean intercept length (MIL) algorithm from the BoneJ plugin in FIJI ${ }^{38}$ to find structural anisotropies related to particle arrangements and morphology. The MIL algorithm computes ellipsoids by projecting vectors from a randomly selected point on a plane, which acts as an origin within the structure. We draw these projected vectors throughout the sample, where we count phase changes for each vector. The mean intercept length for each vector is determined by dividing the vector length by the number of phase changes observed. We generate a point cloud from the large sum of vectors to fit an ellipsoid to the data. We then use this ellipsoid to compare anisotropies across all electrodes. The structural anisotropy is a scalar output from BoneJ, one minus the smallest radii ratio to the largest. Where smaller radii correlate to more 
significant phase changes in the given direction, in equation 11 , isotropic structures will tend to 0 while anisotropic structures will tend to 1 .

\subsection{Heterogeneity Calculations}

In this study, we define two heterogeneity metrics: actual heterogeneity and predicted heterogeneity. We define actual heterogeneity as the absolute value of the difference of the upper and lower quartile ranges of all LRVE values for the given quantity of interest (QOI).

We also expect that the heterogeneity can be predicted by the actual heterogeneity in structural anisotropy using relationships between porosity/solidity and tortuosity/conductivity. Predicted heterogeneity is defined as the difference from propagating the upper and lower quartile of porosity through the Bruggeman approximation. The predicted heterogeneity is done only for conductivity and tortuosity of all directions. Equation 13 describes the predicted heterogeneity

$$
\text { heterogeneity }_{\text {predicted }}=\left|F\left(\epsilon_{Q_{3}}\right)-F\left(\epsilon_{Q_{1}}\right)\right| \text {. }
$$

where the function $F$ is either eq 9 or 10 depending on the QOI, while $\epsilon_{Q_{1}}$ and $\epsilon_{Q_{3}}$ are the lower and upper quartile ranges for porosity, respectively.

\section{Results and Discussion}

In this section, we compare all electrodes from a holistic perspective. We use this view to single out a few scenarios that highlight the morphology and heterogeneity comparisons. We explore the long-range effects and conductivity heterogeneity throughout the electrodes in this study. It is necessary to take a deep dive into how heterogeneities, internal variation, and anisotropies interact 
to assess choosing the electrode material to extract maximum performance from the lithium-ion cell.

\subsection{Tortuosity and Conductivity Anisotropies}

Anisotropies were calculated for both tortuosity and conductivity directly from equation 12. Figure 2 shows that conductivity and tortuosity anisotropy increase at the same rate, exhibited by the dashed line. Figure 2 also shows that as structural anisotropy increases, the effective property anisotropy increases. The correlation between structural and effective property anisotropy is due to the increasing porous pathways, which generates bottlenecks. These bottlenecks restrict potential porous pathways, introducing detours that directly impact tortuosity and conductivity values.

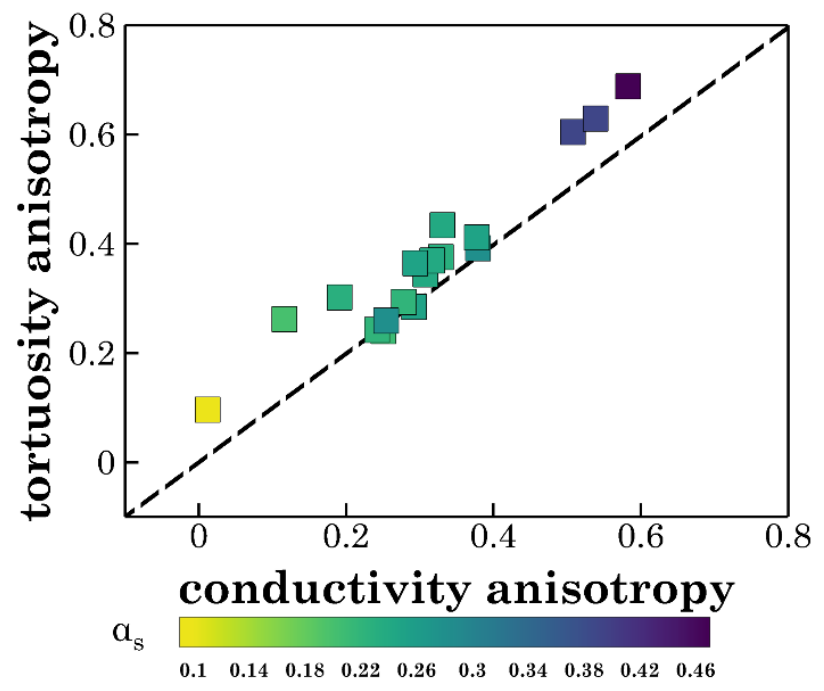

Figure 2. Tortuosity anisotropy, conductivity anisotropy, and structural anisotropy increase similarly for all 18 electrodes. Tortuosity and conductivity anisotropy were calculated using the Bruggeman exponents (shown in eq 12), while structural anisotropy was found through the MIL algorithm. The dashed line has a slope of one, representing that anisotropies of both the properties would be equal and increase at the same rate. 
Each electrode exhibits through-plane dominance in tortuosity, conductivity, and structural anisotropy. Through-plane dominance comes from the exponents in the Bruggeman approximation being larger in the $z$-direction than the $x$ and $y$-directions; this occurs in the electrode structure. The structural anisotropy directly correlates with the particle anisotropy. The lowest and highest structural anisotropies are 0.08 and 0.45 , respectively, which directly correlate to the lowest and highest effective property anisotropies. Electrodes with similar structural anisotropy but different effective property anisotropy could result from porosity differences or differences during the manufacturing process.

Conductive binder domain (CBD) is added in a specified weight percent during manufacturing to produce mechanical rigidity. The composition of $95: 5 \mathrm{wt} \%$ (AM:CBD) is used in most graphite electrodes. ${ }^{8,39}$ The current state-of-the-art electrochemical models use XCT imaging for their geometry; however, $\mathrm{XCT}$ cannot discern the $\mathrm{CBD}$ phase due to low atomic numbers. Thus, this domain is often reconstructed artificially using synthetic models..$^{24,26,40-42}$ The addition of CBD impacts effective properties in cathode materials due to the high volume being added..$^{24,26}$ Graphite and CBD have similar densities; therefore, the volumetric addition is close to 5\% CBD. We performed preliminary studies on randomized LRVEs used in this study and observed, on average, less than 5\% change in tortuosity and conductivity. Therefore, CBD was not added in this study.

\subsection{Impact of Structural Anisotropy on Tortuosity}

Figure 3(a) shows the variation of through-plane tortuosity for each LRVE within an electrode due to its porosity and structural anisotropy. We chose the through-plane over in-plane directions due to the dominant anisotropic behavior for all of the electrodes, as discussed in section 3.1. We 
then use the LRVE values to fit a surrogate model to obtain the contours shown in Figure 3(a). The contours give a more generalized view where data points do not present in one of the 18 electrodes. The contours show that there are no linear relations between structural anisotropy and tortuosity. Through the Bruggeman approximation, we also know that tortuosity and porosity do not have linear relations.
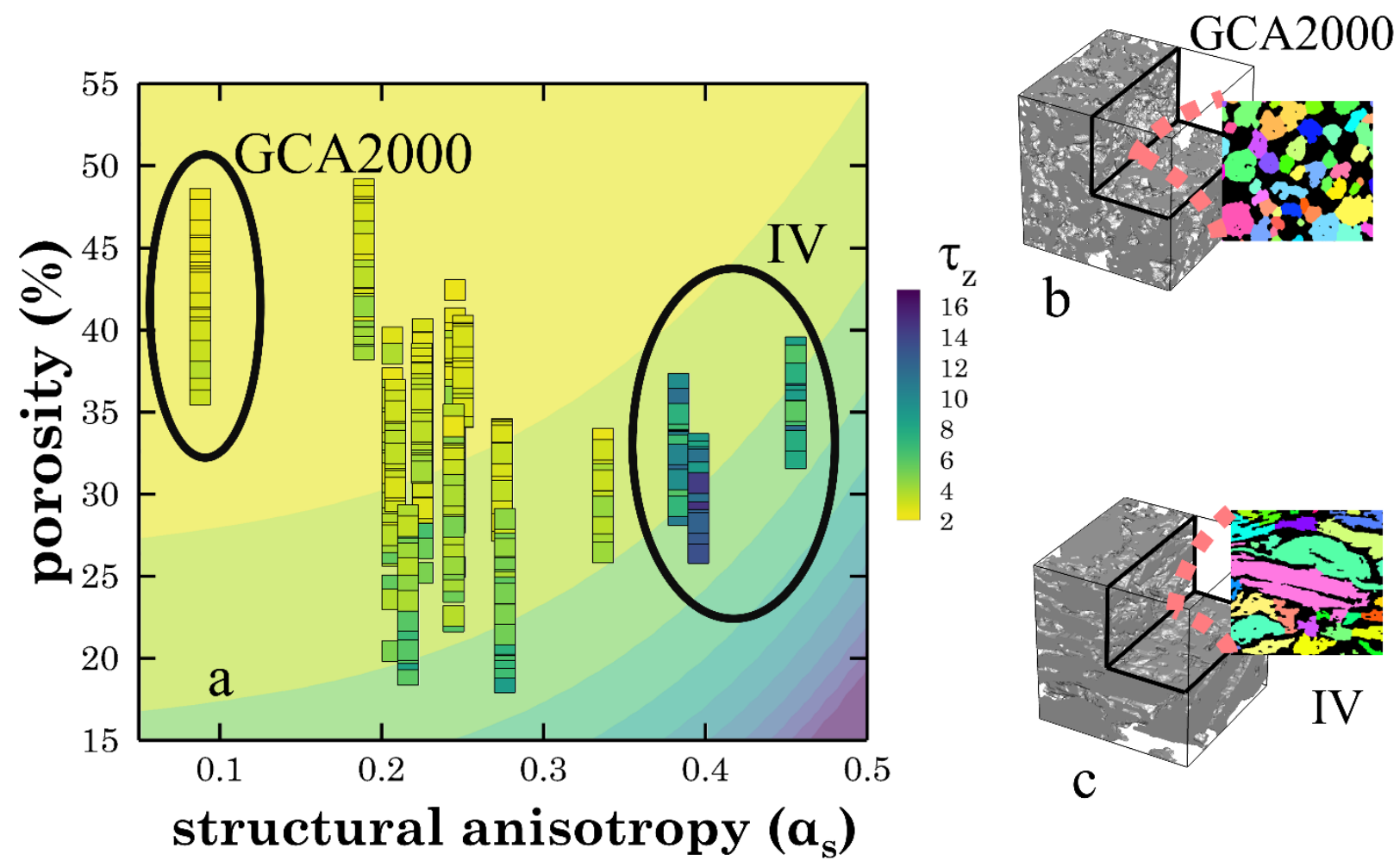

Figure 3. Through-plane tortuosity is dependent on structural anisotropy and porosity. Two electrodes with opposing structural anisotropy are chosen for comparison. (a) Contour plot obtained from a surrogate model, where the contours represent the through-plane tortuosity. The $x$-axis is the structural anisotropy obtained through the MIL algorithm, while the $y$-axis is the porosity. The individual points are individual LRVE values. The contour values shown encompass both the individual points and contours (b) show the structure of GCA2000, along with a slice that has particles identified to show the particle shape. (c) Electrode IV's structure, with a slice of segmented particles to show the shape of the particles. 
Both GCA2000 and electrode IV lie on the two extreme ends of the structural anisotropy spectrum. Figure 3(a) shows where these electrodes lie within the regime map. Both electrodes show similar porosities but electrode IV has greater tortuosity values. GCA2000 and electrode IV have mean porosity values of 42 and $36 \%$, respectively, with mean tortuosity values of $\sim 3$ and 10 . The difference in tortuosity values is due to large structural anisotropies in electrode IV. Figure $3(b, c)$ shows GCA2000 and electrode IV structures with segmented slices from the electrodes. We can visually notice that the particle anisotropy through the slices and structures is much greater in electrode IV compared to GCA2000.

We observe a tighter porosity distribution in electrode IV when comparing the contour lines to the LRVE data in Figure 3(a). As structural anisotropy increases, tortuosity fluctuations among LRVEs begin to become greater. One can capture these fluctuations through the Bruggeman deviation described in equation 11, which captures the influence of particle arrangement. Meaning, if an LRVE has a marginally higher porosity than another, it could result in a higher tortuosity due to bottlenecks stemming from particle arrangement. The derivative of equation 9 can also describe this increase of sensitivity in porosity. The sensitivity is directly dependent on the exponent value given the same porosity.

\subsection{Structural Effect on Heterogeneity}

Comparing electrodes with differing particle morphologies gives insight into how the morphology influences heterogeneity, internal variation, and anisotropies. As mentioned before, electrodes IV and GCA2000 represent the extremes in the particle morphology and structural anisotropy. Therefore, they are prime examples to help unveil the influence of the particle morphology on heterogeneity and internal variation. 
Figure 4(a,b) shows the LRVE data with its respective Bruggeman fit for each electrode. Both electrodes have relatively similar porosity values and with in-plane tortuosity values being similar within electrodes. We see that the through-plane tortuosity in electrode IV is much greater due to the anisotropic nature. There is similar in-plane tortuosity across electrodes. Phase changes from the MIL algorithm occurred at a similar rate for the $x$ and $y$-directions in both electrodes, which results from a 2D isotropic morphology. This helps explain why, given opposing structural anisotropies, we see similar in-plane tortuosity values. Figure 4(a) shows that electrode IV's particles exhibit platelet-like characteristics. Even though electrode IV has a smaller porosity heterogeneity, described by the interquartile range, the tortuosity values have a high propensity to vary much more than GCA2000. We characterize this propensity through the predicted heterogeneity, which is higher for electrode IV due to the magnitude of the tortuosity values.
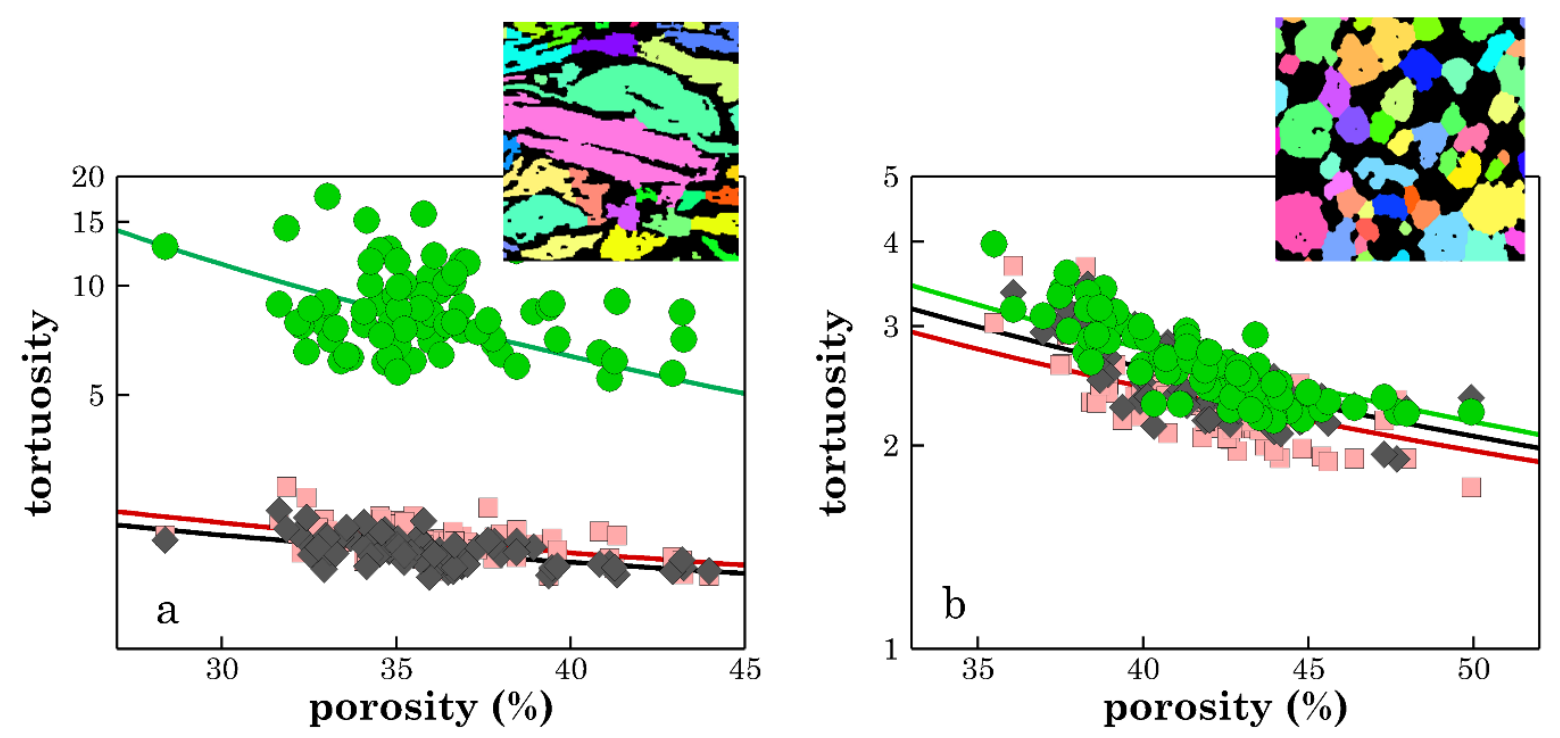

Figure 4. Higher through-plane tortuosity is seen in electrode IV. In-plane tortuosity is similar in both electrodes. (a) Tortuosity of electrode IV in the $x, y$, and $z$ directions is shown in red, gray, and green, respectively. The individual points are the LRVE values, while the solid lines are the Bruggeman approximation that best fit the points. (b) Tortuosity of GCA2000 in $x, y$, and $z$ directions is shown in red, gray, and green, respectively. The individual points are the LRVE values, while the solid lines are the Bruggeman approximation that best fits the points. 
Figure 4 shows how the through-plane tortuosity has a greater heterogeneity in electrode IV than GCA2000. Figure 5 shows the Bruggeman deviation for tortuosity and conductivity of electrode IV and GCA2000. There is a higher average Bruggeman deviation for electrode IV than GCA2000 for both conductivity and tortuosity. The higher Bruggeman deviation explains that electrode IV's tortuosity is more sensitive to particle arrangement. Either the structural anisotropy or high tortuosity could explain why electrode IV has more significant Bruggeman deviations. The spread of deviations is also greater in electrode IV for tortuosity. Figure 5 also shows that conductivity deviation may also be dominated by structural anisotropy. Electrode IV has much lower throughplane conductivity, which forces easy particle disconnection to occur. The higher porosity heterogeneity in GCA2000 could be why there is a larger spread of errors, while the structural anisotropy generates the lower Bruggeman deviation. Does structural anisotropy or porosity heterogeneity influence heterogeneity more? 


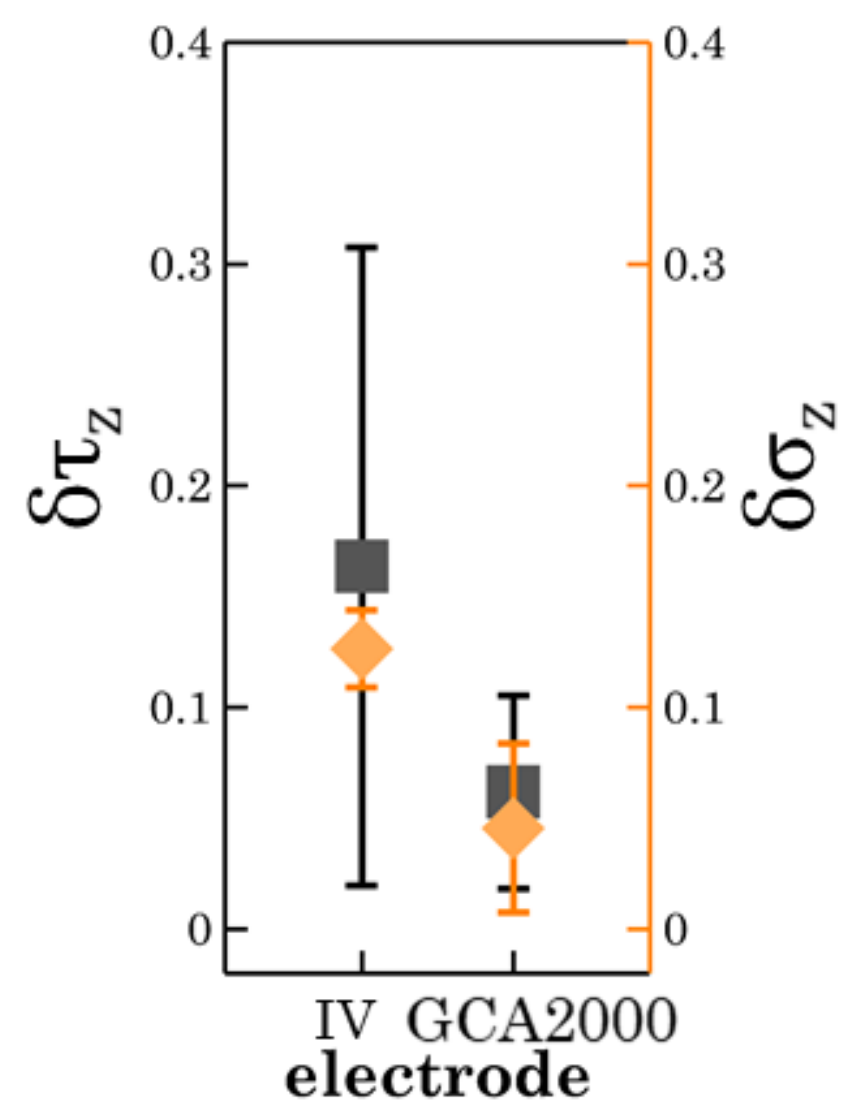

Figure 5. Higher Bruggeman deviation is seen in electrode IV and a greater interquartile range among the LRVE's for tortuosity. Bruggeman deviation in conductivity is higher in electrode IV, but a larger interquartile range is seen in GCA2000. The error bars are the interquartile range of the individual errors $\left(\delta_{L R V E}\right)$.

Figure 6 shows that an electrode with high porosity heterogeneity does not correlate to high predicted tortuosity heterogeneity. This disparity is seen in electrode IV's subdomains having the largest predicted tortuosity heterogeneity while still having relatively low porosity heterogeneity. The discrepancy is because the Bruggeman approximation derivative influences the heterogeneity more than the porosity heterogeneity. 


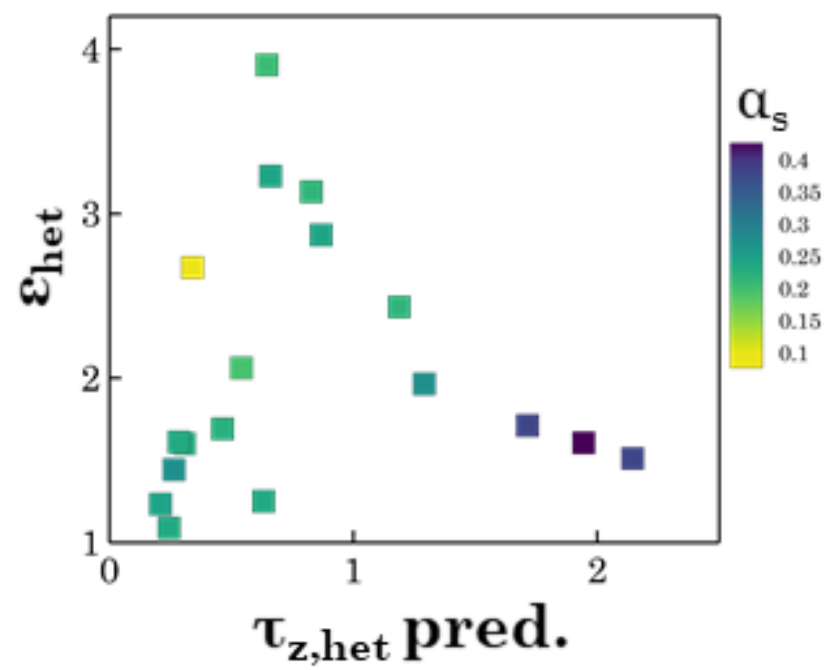

Figure 6. Loose relations between porosity heterogeneity and through-plane tortuosity heterogeneity due to structural anisotropy. The $x$-axis is the predicted heterogeneity for each electrode, the $y$-axis is the porosity heterogeneity of the LRVE's, and the point color is the MIL output.

Since the Bruggeman deviation is calculated at the local porosity for each LRVE, it explains more than just the overall heterogeneity of the electrode. Even though the Bruggeman approximation and porosity heterogeneity account partly for the predicted tortuosity heterogeneity, it does not account for the local variations that the Bruggeman deviation encapsulates. The Bruggeman approximation and porosity heterogeneity only account for sensitivity on the fitted Bruggeman approximation curve. At the same time, the Bruggeman deviation measures how much the LRVEs vary at local porosities. From Figure 5, the Bruggeman deviation is larger where larger averaged through-plane tortuosity occurs. Are there any other factors that influence the deviation, and does this depend on anisotropies?

\subsection{Predicted Heterogeneity with Anisotropic Tortuosity}

Structural anisotropy plays an essential role in determining through-plane heterogeneity. The comparison of electrode IV and GCA2000 gives rise to in-plane tortuosity similarities, and it is a 
natural progression to see how in-plane properties compare across multiple electrodes. We chose three electrodes, Tesla, III, and IV, to probe tortuosity anisotropy. Two of these electrodes show similar porosity values (III and IV), while two share similar structural anisotropies (Tesla and III). Comparisons of tortuosity anisotropies are shown in Figure 7.

Comparing Tesla and electrode III, which have similar structural anisotropy values but different porosities, Figure 7(a-d) shows Tesla's smaller porosity results in higher tortuosity in all three directions. The porosity heterogeneity is also greater in Tesla compared to electrode III. Both the lower porosity and greater porous heterogeneity result in a greater Bruggeman deviation for Tesla. The magnitude of tortuosity in all directions for Tesla results in a larger Bruggeman deviation.

When comparing electrodes III and IV, which have similar porosity values but differing in structural anisotropy, Figure 7(f) shows a much greater Bruggeman deviation in the through-plane direction in electrode IV. The larger deviation is a similar result seen in section 3.3. Even though we have a much greater structural anisotropy in electrode IV, the in -planes in both electrodes show similar Bruggeman deviation values. This similarity reinforces our previous knowledge that the Bruggeman deviations are proportional to the tortuosity values. The similarities of values arise from similar in-plane phase changes seen in both electrodes, resulting in similar in-plane tortuosity.

When comparing electrode IV and Tesla, which share neither porosity nor structural anisotropy values, Figure 7(e and f) shows a much greater Bruggeman deviation occurring for the in-plane direction of Tesla. However, the through-plane of Tesla is marginally larger even though we see smaller tortuosity values. The larger deviation contrasts with what we have seen before, where the larger tortuosity values result in larger Bruggeman deviations. We see more heterogeneity for LRVEs in electrode IV's through-plane tortuosity given by the error bars. Still, the averaged value the LRVE's deviate from the Bruggeman approximation is slightly larger for Tesla. Upon further 
inspection, the much larger porosity heterogeneity plays a factor here. The extreme quarters of tortuosity have the highest deviation values. Since Tesla has a broader porosity distribution, these extreme quarters result in large errors, increasing the deviation.
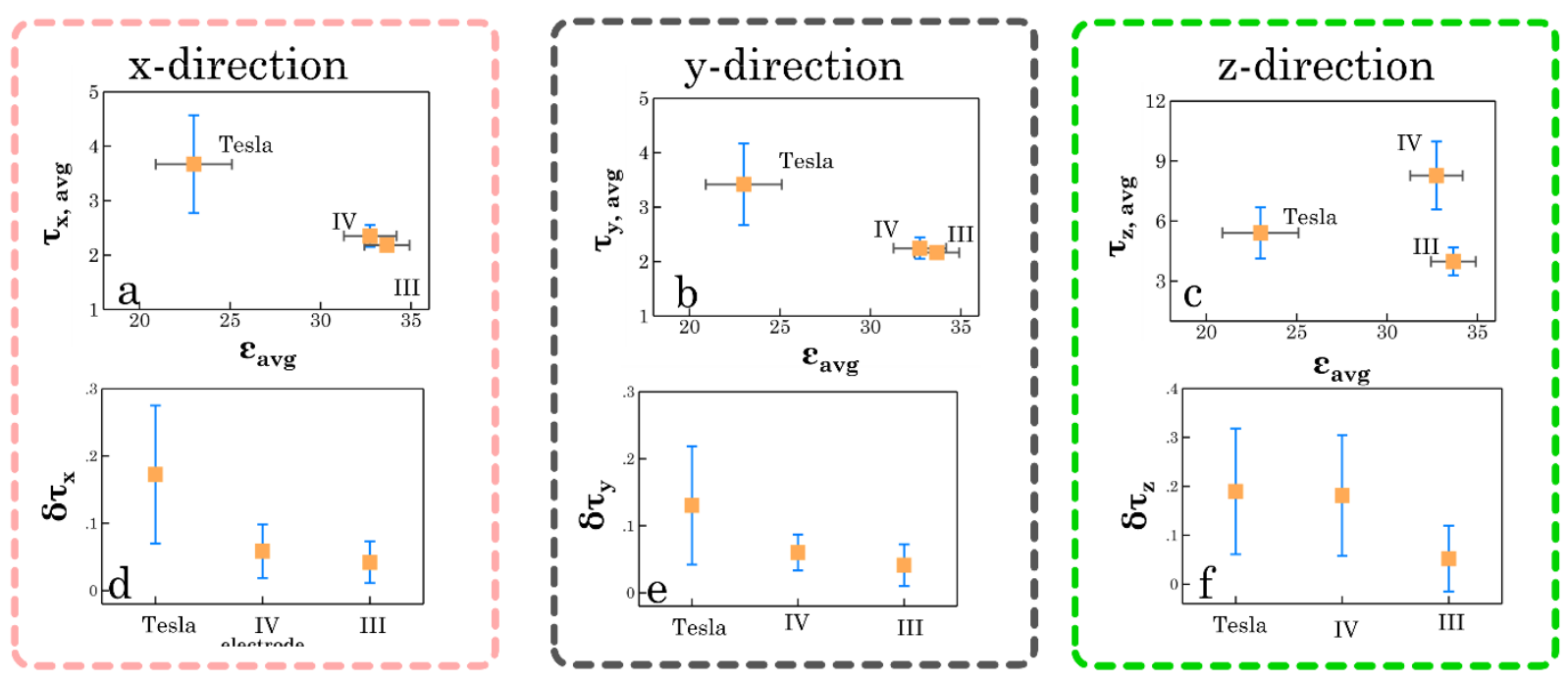

Figure 7. Higher tortuosity results in larger Bruggeman deviation regardless of the direction of interest for tortuosity. (a-c) Averaged tortuosity and porosity for all three electrodes, with the error bars representing the standard deviation of all LVRE's. Each electrode is labeled for all three directions. (d-f) Bruggeman deviation where the error bars represent the standard deviation of all LRVE errors $\left(\delta_{L R V E}\right)$ for all three directions.

Figure 8 shows the $x, y$, and $z$-directions of the interactions with the predicted tortuosity heterogeneity, actual tortuosity heterogeneity, and the Bruggeman deviation for all 18 electrodes. It is seen in all three directions that the actual heterogeneity is larger than the predicted heterogeneity. Also, the deviation is proportional to the difference between the two heterogeneities. Some outliers, such as Tesla seen in Figure 7, may have a higher Bruggeman deviation with lower tortuosity values in the through-plane direction. Larger tortuosity occurs in the through-plane direction due to the influence of structural anisotropy mentioned in section 3.2. This larger tortuosity will propagate to larger predicted and actual heterogeneities, which is seen in Figures 8 and 7(a-c). The reasoning for in-plane directions having nearly no outliers is because 
each electrode exhibited a 2D isotropic structure regarding the in-plane direction, as mentioned in section 3.3. Having different predicted and actual heterogeneities validates the intricacies that particle arrangement influences heterogeneity on a larger scale. Since the deviation does increase with both heterogeneities, particle arrangement is influenced more at high tortuosity values.
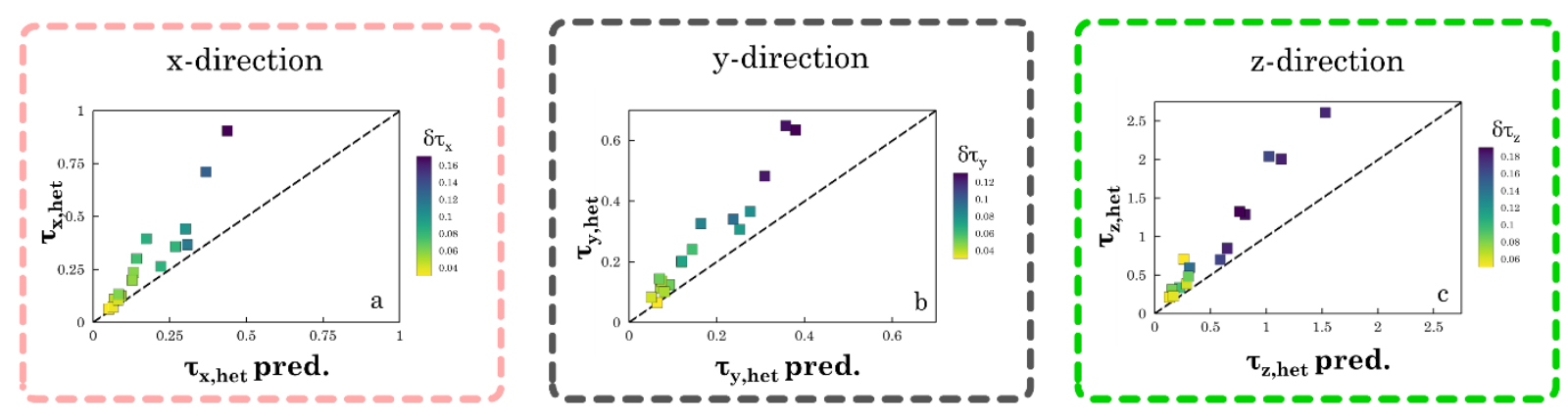

Figure 8. Tortuosity heterogeneity is greater than predicted heterogeneity, where the difference between the two is proportional to the Bruggeman deviation. The larger the predicted heterogeneity, the larger the Bruggeman deviation. (a-c) Predicted vs actual heterogeneity for through-plane tortuosity in each electrode. The color of each point is the Bruggeman deviation for its respective direction. The dashed line, which has a slope of 1 , represents when the predicted heterogeneity is equal to the actual heterogeneity.

\subsection{Particle Packing and Sensitivity}

Figure 6 shows that an increase in porosity heterogeneity does not mean an increase in tortuosity heterogeneity. We show that electrodes with platelets like the particle morphology and densely packed spherical morphology result in sensitive structures. This sensitivity is shown with electrodes IV and Tesla, respectively. These structures resulted in high tortuosity electrodes, which yield a more sensitive arrangement. Figure 9 gives a simplified explanation of why this phenomenon is happening. In electrode IV, represented by Figure $9(a, b)$, the tortuous path is increased dramatically by cutting off one pathway in the platelet-like particle. Compared to electrode III, illustrated by Figure 9(e,f), electrode IV is more likely to have tortuosity changes, 
given a similar porosity range. We can expect platelet particles to show more variability within the structure if choosing between platelet and spherical particles.

The same trend happens when comparing structural anisotropies such as Tesla, shown in Figure 9(c,d), and electrode III. When one packs the structure denser, pathways quickly become bottlenecked. We see that lower porosity ranges will significantly influence the in-planedirections, which also holds for through-plane values given similar structural anisotropies. When comparing through-plane directions, structural anisotropies have a more significant effect on tortuous heterogeneity and Bruggeman deviation. The critical takeaway is that one can observe more significant tortuosity heterogeneity and internal variation in highly anisotropic structures for identical porosity ranges. When we look at a densely packed isotropic structure to a loosely packed anisotropic structure, the in-plane directions dominate the tortuous heterogeneity and internal variation.

Whether packing a structure tightly to get more capacity or choosing platelet-like particles to get more surface area, each choice comes with consequences. It is up to the electrode's creator to choose wisely and weigh the odds to the cell's primary objective. 

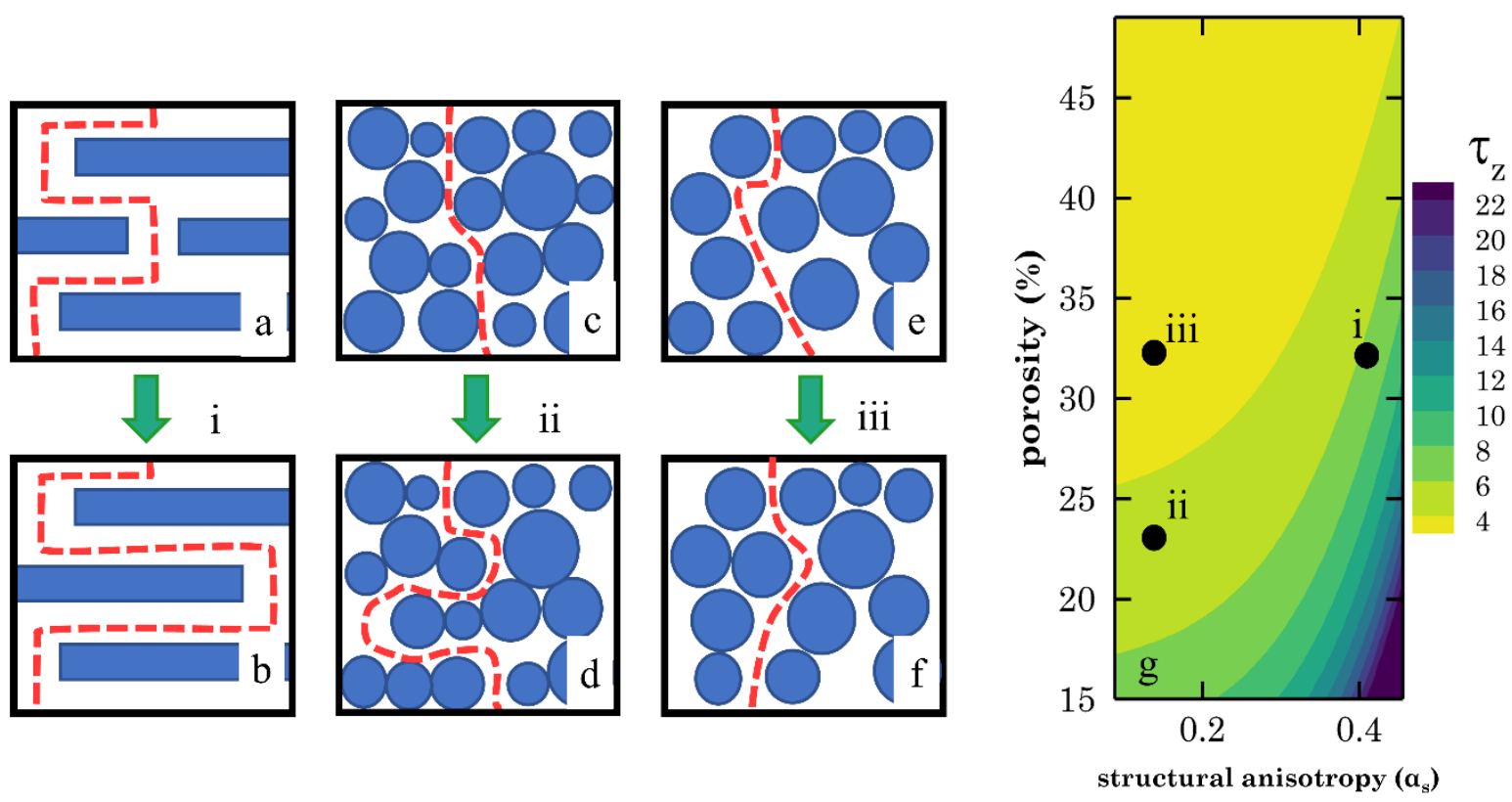

Figure 9. Tortuous pathways can be heavily restricted by small particle movement in low porosity or high structural anisotropy structures resulting in higher sensitive structures. (a, b) Electrode IV, which has a high structural anisotropy. (c, d) Tesla electrode, which has low porosity but a more spherical shape. (e, f) Electrode III, which has similar porosity to IV and a similar structural anisotropy to Tesla. Panel (g) shows where each of these electrodes lies on the regime map.

\subsection{Long-Range Effects}

The previous results sections focused on heterogeneities within a single image sample of an electrode, exploring heterogeneities over $357.5 \mu \mathrm{m}$. However, the numbered electrodes have three subdomains (a-c), which are samples taken from a single electrode sheet at various locations allowing us to explore heterogeneity at larger length scales. We chose electrodes II and IV due to their opposing structural anisotropy but similar porosity ranges. Figure 10 shows the tortuosity heterogeneity and porosity heterogeneity as error bars for each of the three subdomains of both electrodes. Electrode IV has a higher heterogeneity of tortuosity values. There is also a greater porosity heterogeneity in electrode IV.

When comparing the three subdomains for electrodes II and IV, Figure 10 shows a large difference in one of electrode IV's subdomains (IVb). Electrode IVb showed greater structural 
anisotropy, lower porosity, and higher tortuosity. Compared to electrode II, one can see some variation between the subdomains, but they are relatively similar. The differences in electrode IVb could allude to anisotropic particles having more heterogen eous packing over the range of an electrode. The heterogeneity may explain the porosity differences, as well as the structural anisotropy differences in electrode IVb.

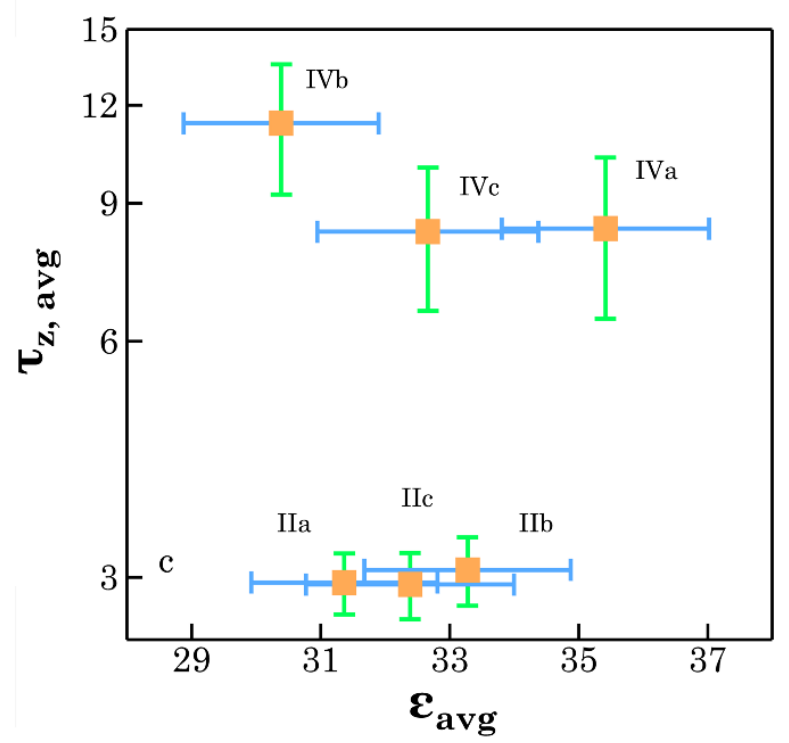

Figure 10. Electrode IV has a greater difference between the three sub-domains compared to electrode II. Each point represents the mean value of all LRVEs, while the error bars represent the standard deviation. Electrode II has a structural anisotropy of $\sim 0.21$, while electrode IV has a structural anisotropy of $\sim 0.43$.

Table 1 shows the $p$-scores from a two-sample Kolmogorov-Smirnov test (K-S test) for the three combinations for through-plane tortuosity. A two-sample test was performed on the QOI distribution of the LRVEs, which gives a K-S statistic and p-score. The Kolmogorov-Smirnov test (K-S test) is a nonparametric test that compares two known distributions. ${ }^{43}$ What is essential to see is thateven in the same material composition, electrode properties could statistically not be similar. Electrode IVa,c shows extreme similarity with a $p$-score of 0.86 . With electrode IVb, we can reject 
the null hypothesis due to low $p$-scores, meaning that electrode IVb is of a different distribution statistically. Electrodes IIa-c all lie within the same distribution if we consider p-scores $>0.01$. Typical p-scores chosen to reject the null-hypothesis are $>0.05$, meaning IIa, IIb, and IIc lie just outside this threshold. The similarities between subdomains with lower tortuosity values or lower structural anisotropy could result in an electrode sheet with less heterogeneity. However, it is essential to see that long-range heterogeneity shows that locations can have statistically different values in the same material composition.

Table 1. Kolmogorov-Smirnov $p$-Score Values Through-Plane Tortuosity Between Interelectrode Subdomains.

\begin{tabular}{|l|l|l|l|}
\hline Electrode & a,b $p$-score & b,c $p$-score & c,a $p$-score \\
\hline II & 0.0203 & 0.015 & 0.2662 \\
\hline IV & $9.95 \times 10^{-8}$ & $6.37 \times 10^{-8}$ & 0.8613 \\
\hline
\end{tabular}

\subsection{Conductivity Heterogeneity}

Figure 11(a-c) shows the predictive conductivity heterogeneity, actual conductivity heterogeneity, and Bruggeman deviation. Predictive conductivity heterogeneity is higher in most cases than actual conductivity heterogeneity. Unlike tortuosity, the deviation is not proportional to the difference between the two. The deviation is loosely proportional to the predicted conductivity heterogeneity in the in-plane directions but not for the through-plane direction. The higher predicted heterogeneity means fewer particle connections is result in larger slopes from the derivative. These larger slopes, like tortuosity, can be representative of the sensitivity of the structure. Some of the differences in heterogeneity trends comparing tortuosity and conductivity could result from the phase of interest related to the quantity of interest. One explanation could be the lack of the pore phase compared to the particle phase, resulting in pathways easily being 
restricted for tortuosity. Another hypothesis could be attributed to the pore phasebeing amorphous, while particles retain their shape. The amorphous nature of the pore phase could mean a higher likelihood of pathway restriction.
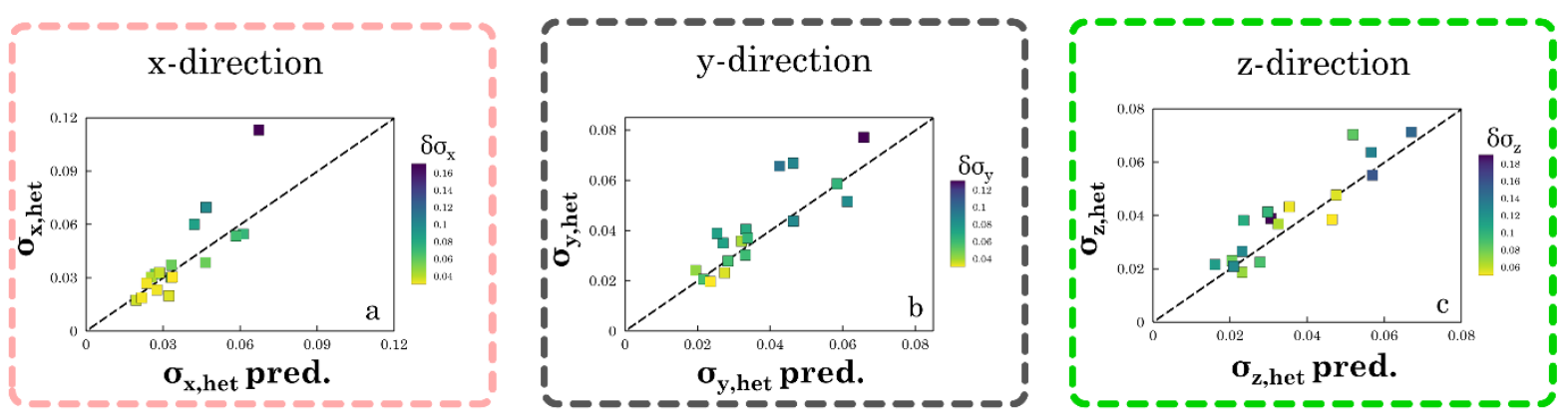

Figure 11. Actual conductivity heterogeneity is greater than predic ted heterogeneity in most cases. The larger the predicted heterogeneity, the larger the Bruggeman deviation. (a-c) Predicted vs actual heterogeneity for through-plane tortuosity in each electrode. The color of each point is the Bruggeman deviation for its respective direction. The dashed line, which has a slope of 1 , represents when the predicted heterogeneity is equal to the actual heterogeneity.

\section{Conclusions}

In this present study, we studied particle morphology and its influence on electrode heterogeneity and anisotropy. We were able to identify multiscale features that impact the sensitivity of effective properties in electrodes that range in particle morphologies. We probed the influence of particle packing on internal variations that result in macroscale heterogeneity. This analysis accounted for 18 open-source tomograms for commercial electrodes.

Our analysis reveals that all 18 electrodes exhibited anisotropy in tortuosity and conductivity, which changed at the same rate. This trend was matched by structural anisotropy, which is heavily influenced by the particle morphology. Tortuosity, conductivity, and structural anisotropy were through-plane dominant in every electrode. We elucidated that the particle morphology influences internal variation by showing that platelet-like particles gave more significant internal variation 
within the LRVEs. This particle morphology also resulted in larger through-plane tortuosity, but in-plane directions were similar because of similar porosity values.

A regime map was created using individual LRVEs of all 18 electrodes. This regime captured physical phenomena seen in our electrodes and known phenomena seen in the literature. The regime map allowed easy comparison and helped elucidate ideas on the sensitivity of an electrode's structure. Using the regime map can be helpful when choosing electrode parameters during manufacturing. We see that platelet-like particles for long-range effects gave more significant variation and heterogeneity than a calendared spherical particle. This long-range effect results from the regime in which the platelet structure resides, lending to a more sensitive arrangement. The particle arrangement led to showing that porosity heterogeneity does not correlate to tortuous heterogeneity.

A structure may be homogeneous regarding porosity, but due to the particle packing, tortuosity can remain heterogeneous. An electrode with a platelet-like particle morphology will exhibit more significant internal variation and heterogeneity for a given porosity, but overall tortuosity values in all directions are a greater influence. This influence is due to the in-plane directions dominating the resulting variability significantly. The in-plane directions are essential due to electrochemical interactions occurring in all Cartesian directions upon cell cycling. Structures with highly tortuous paths will lend to more sensitive electrode structures. This sensitivity relates directly to internal variations and the ability of heterogeneities to arise, making electrode manufacturers perform a cost-benefit analysis to create safer, more reliable electrodes.

\section{Acknowledgments}

The authors used the electrode XCT images from the open-source data provided by Dr. Vanessa Wood's group. This paper describes objective technical results and analysis. Any subjective views 
or opinions that might be expressed in the article do not necessarily represent the views of the U.S. Department of Energy or the United States Government. Supported by the Laboratory Directed Research and Development program at Sandia National Laboratories, a multimission laboratory managed and operated by National Technology and Engineering Solutions of Sandia, LLC., a wholly owned subsidiary of Honeywell International, Inc., for the U.S. Department of Energy's National Nuclear Security Administration under contract DE-NA-0003525.

\section{References}

(1) Goodenough, J. B.; Park, K.-S. The Li-Ion Rechargeable Battery: A Perspective. J. Am. Chem. Soc. 2013, 135 (4), 1167-1176. https://doi.org/10.1021/ja3091438.

(2) Choi, J. W.; Aurbach, D. Promise and Reality of Post-Lithium-Ion Batteries with High Energy Densities. Nat. Rev. Mater. 2016,1, 16013.10.1038/natrevmats.2016.13

(3) Yoshio M.; Wang, H.; Fukuda, K.; Umeno, T.; Abe, T.; Ogumi, Z. Improvement of Natural Graphite as a Lithium-Ion Battery Anode Material, from Raw Flake to Carbon-Coated Sphere. J. Mater. Chem. 2004, 14 (11), 1754-1758. https://doi.org/10.1039/b316702j.

(4) Colclasure, A. M.; Dunlop, A. R.; Trask, S. E.; Polzin, B. J.; Jansen, A. N.; Smith, K. Requirements for Enabling Extreme Fast Charging of High Energy Density Li-Ion Cells While Avoiding Lithium Plating. J. Electrochem. Soc. 2019, 166 (8), A1412-A1424. https://doi.org/10.1149/2.0451908jes.

(5) Buqa, H.; Goers, D.; Holzapfel, M.; Spahr, M. E.; Novák, P. High Rate Capability of Graphite Negative Electrodes for Lithium-Ion Batteries. J. Electrochem. Soc. 2005, 152 (2), A474. https://doi.org/10.1149/1.1851055.

(6) Qie, L.; Chen, W. M.; Wang, Z. H.; Shao, Q. G.; Li, X.; Yuan, L. X.; Hu, X. L.; Zhang, W. X.; Huang, Y. H. Nitrogen-Doped Porous Carbon Nanofiber Webs as Anodes for Lithium 
Ion Batteries with a Superhigh Capacity and Rate Capability. Adv. Mater. 2012, 24 (15), 2047-2050.https://doi.org/10.1002/adma.201104634.

(7) Nanda, J.; Remillard, J.; O’Neill, A.; Bernardi, D.; Ro, T.; Nietering, K. E.; Go, J. Y.; Miller, T. J. Local State-of-Charge Mapping of Lithium-Ion Battery Electrodes. Adv. Funct. Mater. 2011, 21 (17), 3282-3290. https://doi.org/10.1002/adfm.201100157.

(8) Ebner, M.; Geldmacher, F.; Marone, F.; Stampanoni, M.; Wood, V. X-Ray Tomography of Porous, Transition Metal Oxide Based Lithium Ion Battery Electrodes. Adv. Energy Mater. 2013, 3 (7), 845-850. https://doi.org/10.1002/aenm.201200932.

(9) Henini, M. Scanning Electron Microscopy: An Introduction. III-Vs Rev. 2000, 13 (4), 4044. https://doi.org/10.1016/S0961-1290(00)80006-X.

(10) Hutzenlaub, T.; Asthana, A.; Becker, J.; Wheeler, D. R.; Zengerle, R.; Thiele, S. FIB/SEMBased Calculation of Tortuosity in a Porous LiCoO2 Cathode for a Li-Ion Battery. Electrochem. commun. 2013, 27, 77-80. https://doi.org/10.1016/j.elecom.2012.11.006.

(11) Hawley, W. B.; Li, J. Electrode Manufacturing for Lithium-Ion Batteries-Analysis of Current and next Generation Processing. J. Energy Storage 2019, 25, 100862. https://doi.org/10.1016/j.est.2019.100862.

(12) Mistry, A.; Mukherjee, P. P. Deconstructing Electrode Pore Network to Learn Transport Distortion. Phys. Fluids 2019, 31 (12), 122005. https://doi.org/10.1063/1.5124099.

(13) Mistry, A.; Smith, K.; Mukherjee, P. P. Stochasticity at Scales Leads to Lithium Intercalation Cascade. ACS Appl. Mater. Interfaces 2020, 12 (14), 16359-16366. https://doi.org/10.1021/ACSAMI.9B23155.

(14) Mao, C.; Ruther, R. E.; Li, J.; Du, Z.; Belharouak, I. Identifying the Limiting Electrode in Lithium Ion Batteries for Extreme Fast Charging. Electrochem. commun. 2018, 97, 37-41. 
https://doi.org/10.1016/j.elecom.2018.10.007.

(15) Mistry, A.; Juarez-Robles, D.; Stein, M.; Smith, K.; Mukherjee, P. P. Analysis of LongRange Interaction in Lithium-Ion Battery Electrodes. J. Electrochem. Energy Convers. Storage 2016, 13 (3). https://doi.org/10.1115/1.4035198.

(16) Argonne National Laboratory. X-CEL: EXtreme Fast Charge Cell Evaluation of LithiumIon Batteries Report Period: January - June 2018; 2018.

(17) Kehrwald, D.; ... P. S.-J. of T.; 2011, undefined. Local Tortuosity Inhomogeneities in a Lithium Battery Composite Electrode. Electrochem. Soc. 2011, 158 (12), A1393. https://doi.org/10.1149/2.079112jes.

(18) Forouzan, M. M.; Mazzeo, B. A.; Wheeler, D. R. Modeling the Effects of Electrode Microstructural Heterogeneities on Li-Ion Battery Performance and Lifetime. $J$. Electrochem. Soc. 2018, 165(10), A2127-A2144. https://doi.org/10.1149/2.1281809jes.

(19) Abboud, A. W.; Dufek, E. J.; Liaw, B. Communication-Implications of Local Current Density Variations on Lithium Plating Affected by Cathode Particle Size. J. Electrochem. Soc. 2019, 166(4), A667-A669. https://doi.org/10.1149/2.0711904jes.

(20) Müller, S.; Eller, J.; Ebner, M.; Burns, C.; Dahn, J.; Wood, V. Quantifying Inhomogeneity of Lithium Ion Battery Electrodes and Its Influence on Electrochemical Performance. $J$. Electrochem. Soc. 2018, 165 (2), A339-A344. https://doi.org/10.1149/2.0311802jes.

(21) Cooper, S. J.; Eastwood, D. S.; Gelb, J.; Damblanc, G.; Brett, D. J. L.; Bradley, R. S.; Withers, P. J.; Lee, P. D.; Marquis, A. J.; Brandon, N. P.; Shearing, P. R. Image Based Modelling of Microstructural Heterogeneity in LiFePO 4 Electrodes for Li-Ion Batteries. $J$. Power Sources 2014, 247, 1033-1039. https://doi.org/10.1016/j.jpowsour.2013.04.156.

(22) Hein, S.; Latz, A. Influence of Local Lithium Metal Deposition in 3D Microstructures on 
Local and Global Behavior of Lithium-Ion Batteries. Electrochim. Acta 2016, 201,354365. https://doi.org/10.1016/j.electacta.2016.01.220.

(23) Krizhevsky, A.; Sutskever, I.; Hinton, G. E. ImageNet Classification with Deep Convolutional Neural Networks. Commun. ACM 2017, 60 (6), 84-90. https://doi.org/10.1145/3065386.

(24) Trembacki, B. L.; Mistry, A. N.; Noble, D. R.; Ferraro, M. E.; Mukherjee, P. P.; Roberts, S. A. Editors' Choice-Mesoscale Analysis of Conductive Binder Domain Morphology in Lithium-Ion Battery Electrodes. J. Electrochem. Soc. 2018, 165 (13), E725-E736. https://doi.org/10.1149/2.0981813jes.

(25) Cai, L.; An, K.; Feng, Z.; Liang, C.; Harris, S. J. In-Situ Observation of Inhomogeneous Degradation in Large Format Li-Ion Cells by Neutron Diffraction. J. Power Sources 2013, 236, 163-168. https://doi.org/10.1016/j.jpowsour.2013.02.066.

(26) Mistry, A. N.; Smith, K.; Mukherjee, P. P. Secondary-Phase Stochastics in Lithium-Ion Battery Electrodes. ACS Appl. Mater. Interfaces 2018, 10 (7), 6317-6326. https://doi.org/10.1021/acsami.7b17771.

(27) Newman, J.; Tiedemann, W. Porous-electrode Theory with Battery Applications. AIChE Journal. 1975, pp 25-41. https://doi.org/10.1002/aic.690210103.

(28) Roberts, S. A.; Brunini, V. E.; Long, K. N.; Grillet, A. M. A Framework for ThreeDimensional Mesoscale Modeling of Anisotropic Swelling and Mechanical Deformation in Lithium-Ion Electrodes. J. Electrochem. Soc. 2014, 161 (11), F3052-F3059. https://doi.org/10.1149/2.008141 ljes.

(29) Ebner, M.; Chung, D. W.; García, R. E.; Wood, V. Tortuosity Anisotropy in Lithium-Ion Battery Electrodes. $A d v$ Energy Mater. 2014, 4 
https://doi.org/10.1002/aenm.201301278.

(30) Yoshio, M.; Mukai, T.; Ohno, H.; Kato, T. One-Dimensional Ion Transport in SelfOrganized Columnar Ionic Liquids. J. Am. Chem. Soc. 2004, 126 (4), 994-995. https://doi.org/10.1021/ja0382516.

(31) Harris, S. J.; Lu, P. Effects of Inhomogeneities -Nanoscale to Mesoscale -on the Durability of Li-Ion Batteries. J. Phys. Chem. C 2013, 117 (13), 6481-6492. https://doi.org/10.1021/jp311431z.

(32) Pietsch, P.; Ebner, M.; Marone, F.; Stampanoni, M.; Wood, V. Determining the Uncertainty in Lithium Ion Battery Micro-Structural Parameters Extracted from Tomographic Data. In Chemistry and Materials for Lead-Based Batteries Symposium 2018, Held at AABC Europe 2018; 2018; pp 391-392.

(33) Krygier, M. C.; LaBonte, T.; Martinez, C.; Norris, C.; Sharma, K.; Collins, L. N.; Mukherjee, P. P.; Roberts, S. A. Quantifying the Unknown Impact of Segmentation Uncertainty on Image-Based Simulations. Nat. Commun. 2021, 12 (1). https://doi.org/10.1038/s41467-021-25493-8.

(34) Trembacki, B.; Mistry, A.; ... D. N.-J. of T.; 2018, U. Mesoscale Analysis of Conductive Binder Domain Morphology in Lithium-Ion Battery Electrodes. jes.ecsdl.org.

(35) Lomax, H.; Pulliam, T.; Zingg, D.; Kowalewski, T. Fundamentals of Computational Fluid Dynamics. Appl. Mech. Rev. 2002, 55 (4), B61. https://doi.org/10.1115/1.1483340.

(36) Saad, Y. Iterative Methods for Sparse Linear Systems; 2003. https://doi.org/10.1137/1.9780898718003.

(37) Bruggeman, D. A. G. Berechnung Verschiedener Physikalischer Konstanten von Heterogenen Substanzen. I. Dielektrizitätskonstanten Und Leitfähigkeiten Der Mischkörper 
Aus Isotropen Substanzen. Ann. Phys. 1935, $416 \quad$ (7), 636-664. https://doi.org/10.1002/andp.19354160705.

(38) Doube, M.; Klosowski, M. M.; Arganda-Carreras, I.; Cordelières, F. P.; Dougherty, R. P.; Jackson, J. S.; Schmid, B.; Hutchinson, J. R.; Shefelbine, S. J. BoneJ: Free and Extensible Bone Image Analysis in ImageJ. Bone 2010, 47 (6), 1076-1079. https://doi.org/10.1016/j.bone.2010.08.023.

(39) Zielke, L.; Hutzenlaub, T.; Wheeler, D. R.; Chao, C. W.; Manke, I.; Hilger, A.; Paust, N.; Zengerle, R.; Thiele, S. Three-Phase Multiscale Modeling of a LiCoO2 Cathode: Combining the Advantages of FIB-SEM Imaging and X-Ray Tomography. Adv. Energy Mater. 2015, 5 (5), 1401612. https://doi.org/10.1002/aenm.201401612.

(40) Trembacki, B. L.; Noble, D. R.; Brunini, V. E.; Ferraro, M. E.; Roberts, S. A. Mesoscale Effective Property Simulations Incorporating Conductive Binder. J. Electrochem. Soc. 2017, 164 (11), E3613-E3626. https://doi.org/10.1149/2.0601711jes.

(41) Zielke, L.; Hutzenlaub, T.; Wheeler, D. R.; Manke, I.; Arlt, T.; Paust, N.; Zengerle, R.; Thiele, S. A Combination of X-Ray Tomography and Carbon Binder Modeling: Reconstructing the Three Phases of LiCoO2Li-Ion Battery Cathodes. Adv. Energy Mater. 2014, 4 (8), 1-6. https://doi.org/10.1002/aenm.201301617.

(42) Srivastava, I.; Bolintineanu, D. S.; Lechman, J. B.; Roberts, S. A. Controlling Binder Adhesion to Impact Electrode Mesostructures and Transport. ACS Appl. Mater. Interfaces 2020, 12 (31), 34919-34930. https://doi.org/10.1021/acsami.0c08251.

(43) Massey, F. J. The Kolmogorov-Smirnov Test for Goodness of Fit. J. Am. Stat. Assoc. 1951, 46 (253), 68-78. https://doi.org/10.1080/01621459.1951.10500769. 
Table of Content (TOC) Figure

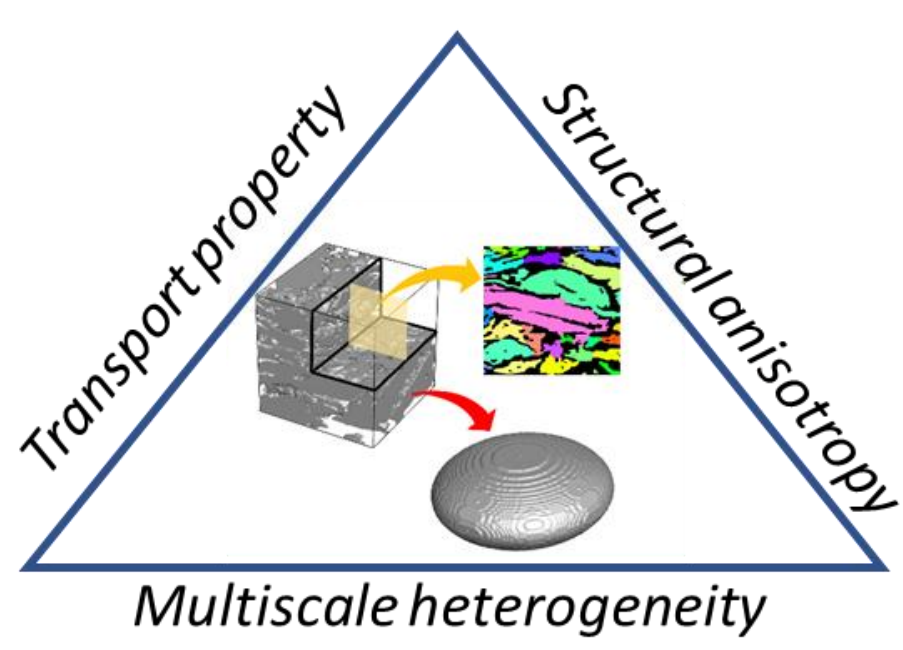

